\title{
Face to face and telephone interviews as competing yet complementary methods to elicit information
}

\author{
B. Barabino ${ }^{1}$, E. Deiana ${ }^{2} \&$ P. Tilocca ${ }^{3}$ \\ ${ }^{1}$ Department of Civil Engineering, Environment and Archaeology, \\ Cagliari University, Italy \\ ${ }^{2}$ Technomobility Ltd, Cagliari, Italy \\ ${ }^{3}$ CTM SpA, Cagliari, Italy
}

\begin{abstract}
Face to face (intercept/at home) and telephone surveys have been thoroughly investigated in the service industry literature. Regardless of this, the so far limited studies in the public transportation sector do not state clearly which method yields the better results. Besides, evaluating the more appropriate datacollection mode is a complex task, due to the many constraints imposed (e.g. time, budget, and so on). This paper will critically compare the results of two different surveys conducted in 2012 within the Cagliari metropolitan area by the local transit agency CTM, with the chief goal to evaluate which of the two administration methodologies produces the more reliable results. A total of 500 fairly long interviews with CTM's users and 714 short interviews with non users were collected through the telephone survey, whilst the on-board intercept survey resulted in 3173 interviews. The most significant strength of the telephone methodology referred to the possibility to reach non users and to elicit information on the reasons behind their decision to rely solely on private transport. On the other hand, the main strongpoint of the on-board methodology has regarded the high representativeness of the data collected, rich in information and qualitatively reliable. Therefore, the two approaches do not exclude each other, with on-board and telephone surveys that might be targeted at users and non users, respectively. Finally, this paper is expected to improve the knowledge of existing and likely demand for bus operators and offers valuable lessons for public transport agencies worldwide.
\end{abstract}

Keywords: urban transport, survey methods, transit intercept and telephone surveys, data quality. 


\section{Introduction}

Local transit agencies are nowadays placing more importance on their customers' needs and desires, in an attempt to stay afloat and gain new shares in a transport market characterized by higher levels of competition and reduced entry barriers to competitors. Moreover, budgetary constraints require an optimization of the service offered, which should mirror as much as possible its demand characteristics, so to prevent resources from being wasted and encourage their channelling towards core aspects of the service. These two different but intertwined goals, attracting new customers through the optimization of the limited resources available, require for transport operators the acquisition of a critical mass of information previously overlooked in favour of an approach heavily skewed towards the sole quantitative supply of the transport service.

The need for more information has brought a renewed interest in surveys on travel behaviours and customer satisfaction, besides their different modes of administration and the validity/reliability of the data collected adopting alternative data gathering approaches. This study will critically compare a telephone and an on-board intercept survey conducted in the metropolitan area of Cagliari, Italy, to assess which of the two can help achieving better results and, eventually, lead the local transport operator towards the improvements of its customer oriented policies.

\section{Survey types in the service industry}

Modes of surveys administration vary significantly in social sciences, with effects on costs, sampling accuracy, response rate, respondent bias, data quality (Bowling [1]). Historically, telephone, postal and at home/intercept surveys represented the prevailing modes of data gathering, whilst the emergence of the world-wide web has brought by an increased use of self administered Internet surveys. The four methodologies, all widely used in the service industry, present each pros and cons. At home/intercept surveys represent the most expensive methodology, with Bradburn and Sudman [2] suggesting their overall cost to be twice as high as the one associated to telephone surveys and up to ten times the one of mail surveys. A further problem of this methodology relates to those biases (social desirability, acquiescence and so forth) associated to the interviewer-respondent interactions during the questionnaire administration phase. However, intercept surveys are also characterized by enhanced sampling accuracies and high response rates, particularly when compared to self administered methods.

Both telephone and postal mail surveys suffer from sampling coverage problems, with the former methodology that is likely to exacerbate this weakness given the progressive abandoning of land lines in favour of mobile telephones, a trend that is particularly relevant among the youngest (the target of reference for most investigations). As for the latter, a recent paper comparing 39 studies published over the last 10 years has shown an average response rate of just $44 \%$ (Shi and Fan [3]). Self administered Internet surveys, despite being the least 
expensive among the four, still suffer from sampling coverage problems and low response rates (Lozar Manfreda et al. [4]).

With reference to data quality issues, we point out the lack of an agreement over which of the methodologies currently implemented can provide companies with the most reliable and accurate pieces of information. Whilst some studies claim that face-to-face surveys provide richer and more reliable data (Bonnel and Le Nir [5]), such views are challenged by authors who believe that other techniques can equally provide good quality data, especially when costs associated to surveys implementation are taken into consideration (Sturges and Hanrahan [6]). However, it is important to point out that no single data collection methodology is free from weaknesses, a feature that has brought some researchers to advocate the concurrent adoption of different data collection techniques (de Leeuw and Hox [7]).

\subsection{The literature on travel surveys}

Household travel surveys for the investigation of mobility features and patterns first appeared in 1965, with the UK National Travel survey. Since then, they have been adopted in developed countries and, from the early 2000s onwards, in some developing economies such as South Africa, Brazil and Chile. Differences are retraceable in their periodicities: annual in countries such as the UK and the Netherlands, irregular in France and Germany and non-existent in Spain and Belgium (Endemann [8]). Stopher [9] shows that differences are also retraceable in their administration modes, ranging from face-to-face (the UK and South Africa) to telephone interviewing (Canada, the US) and self completion postal surveys (Germany and the Netherlands). Travel surveys are being increasingly conducted at metropolitan area level too, again with different periodicities and administration modes, from face-to-face (Sidney, Adelaide) to telephone interviews (Toronto, Chicago) and self-administered questionnaires (Melbourne, Hobart) (Inbakaran and Kroen [10]). Despite being widespread among transit agencies, the present literature on survey methods in public transportation is not as compelling as in other service industry sectors. Some authors argue that in person interviews yield highest quality data, response rates and sampling coverage, despite being significantly more expensive than telephone and, especially, mail and internet modes (Sharp and Murakami [11]). However, such view is not shared by everyone, as the feasibility of a survey over the others depends significantly on elements related to the survey objectives and the cultural and social context pertaining to each one (Bonnel [12]). A classical example is represented by the Netherlands, where the switch from a telephone to a mail administered investigation methodology, decided in 1998, produced an increase in the response rates (Moritz and Brög [13]).

Practical considerations also have a role to play in deciding which methodologies are most suited. In the US face to face/at home interviews have been increasingly substituted by telephone investigations because of security reasons (Stopher [9]). Coherently with other areas in the service industry, a relevant school of thought believes that the adoption of mixed mode surveys might help in obtaining better quality data and increase the response rates 
(Morris and Adler [14]), despite the several challenges linked to data comparability issues (Bonnel [12]; Bayard and Bonnel [15]).

\subsection{The literature on customer satisfaction surveys in public transport}

Customer satisfaction analyses in public transport have recently received much attention, with several studies approaching the topic by means of qualitative and, most often, quantitative investigations. Intercept surveys, both on-board and at selected points, represent the most widely adopted approach to elicit information on customers satisfaction (Eboli and Mazzulla [16]; Lai and Chen [17]; Thompson and Schofield [18]). Examples of surveys conducted over the telephone are available too, despite possible negative effects on data quality resulting from the lack of a contact between the interviewer and the respondent (Fellesson and Friman [19]). Data on customers satisfaction have also been elicited through mail surveys, with Friman et al. [20]) investigating the main factors responsible for feelings of satisfaction and dissatisfaction among Swedish users. Too and Earl [21] and Cantwell et al. [22] implemented web surveys to assess the degree of customer satisfaction towards the provision of public transport in the communities of Varsity Lakes (Australia) and Dublin (Ireland). In both studies, low costs linked to the specific methodology adopted and the widespread use of the internet were used to justify the data collection method chosen, despite the risk of low response rates deriving from the self administered nature of the questionnaires developed.

Despite limited in number, some attempts to mix different methodologies have been advanced, with Tyrinopoulos and Aifadopoulou [23] integrating interviews, on site surveys and mystery shopping measures to assess the levels of quality and performance attained by some major Greek public transport operators. Finally, some qualitative studies based on in depth personal interviews have been presented to provide a deeper understanding of the factors impacting travel behaviours and transport mode choice (Beirão and Sarsfield Cabral [24]).

\section{Our methodology}

Two surveys were conducted in the city of Cagliari during March and October 2012, respectively. They were taken in different periods because of practical reasons, with the local transport agency conducting a telephone survey since 1998 and a biannual intercept survey from 2011 onwards, both designed to elicit socio-demographic, travel and customer satisfaction information. It is important to stress that both surveys have been conducted during the winter service period, thus ensuring a better comparability of the data collected.

The on-board intercept survey was conducted in March, lasted two weeks and resulted in 3173 interviews completed out of 3659 contacts established in the 29 routes investigated. The telephone survey was conducted in October, lasted three weeks and resulted in 500 interviews completed out of the 1379 contacts established. However, this interview could be completed only by local transit agency customers who agreed to take part in the survey, whilst non users were 
administered a shorter version of 6 questions designed to gather information pertaining to the motives for the non use of the local transport service, the possible past use of it and/or of alternative public carriers among the others.

\subsection{Sampling procedures}

A sampling plan with the number of interviews to complete at route level was drawn up for the on-board intercept survey. The minimum number of interviews per route was dependent on the routes frequency, which, in turn, were determined according to the average load factors recorded over the previous years. Hence, high frequency routes were privileged, with sampling errors lower than $10 \%$ for 8 out of the 9 most used routes. Furthermore, sampling errors were kept lower than $20 \%$ even for medium and low frequency routes, with two exceptions pertaining to extremely marginal routes in terms frequency and load factors. Overall, 9.36\% of the on-board passengers counted during the survey were interviewed, with a better percentage coverage of low frequency/load factors routes.

Because of the local transit agency's sampling routines, 500 telephone interviews were required, with the first 100 to be completed among some of the 2011 telephone survey participants and the remaining 400 to be randomly carried out among listed private numbers owners. In order to ensure a proper coverage of the local public transport system users, the number of interviews to be completed in each of the 8 municipalities served by the transit agency was proportional to the demographic weight hold by each municipality in the overall area served (e.g. in case a municipality comprised the $10 \%$ of the population living in the area of study, 50 out of the 500 interviews had to be completed among its residents).

\subsection{Field surveys}

The on-board intercept survey took place during weekdays, between 7AM-7PM. 16 samplers were employed and distributed in teams variable in size between 1 and 3 components, depending on the on-board crowding conditions of each particular route investigated. The questionnaire was administered using a simple random sampling procedure characterized by the equal probability of every onboard passenger to be interviewed. Pilot tests previously conducted and the questionnaire length suggested the need to avoid self administered surveys, despite the higher costs associated to the use of a team of interviewers. Therefore, a personal interview was preferred over a self administered one with the aims to minimize the possible risk of questions misunderstanding, non response errors and maximize the data quality.

The telephone survey took place during weekdays too, with interviews conducted between 10AM-2PM and 4PM-9PM. 4 interviewers, working from workstations installed in the local transit agency headquarters, were used to conduct the survey. The sample to survey was randomly extracted from the telephone directory, with 4 interviews to be completed every 3 pages according to the following distribution: $1-1-2,1-2-1$ and $2-1-1$ for the first, the second and 
the third three pages respectively. The process had to be reiterated until the number of complete interviews scheduled per single municipality was reached.

\subsection{Questionnaires}

The on-board intercept questionnaire was divided in two parts: a travel and a customer satisfaction survey, with the former encompassing few preliminary questions designed to gather socio-demographic information. The travel survey was comprised of closed ended questions aimed at showing information such as the main socio demographic characteristics of the panel interviewed, frequency of use, prevailing trips reasons, main reasons for using the bus transport system. The customer satisfaction survey was comprised of a modified SERVQUAL methodology, aimed at discovering the existing gaps between customers' quality expectations and perceptions and structured following the one recently proposed by Barabino et al. [25].

Likewise, the telephone questionnaire was comprised of a travel and a customer satisfaction survey based on the same modified SERVQUAL methodology. However, the two questionnaires were slightly different, as the travel survey included also a question on the main trips origins/destinations, whilst the customer satisfaction survey included fewer questions.

\section{Results}

This section of the study presents a comparison of the main summary results emerged from the two methodologies implemented, with the goal to disclose whether different administration modes impact on the socio-demographic characteristics of the customers surveyed, their travel habits, their level of satisfaction and expectation with regard to specific service attributes. More importantly, this section will critically analyze and compare strengths and weaknesses as emerged following the implementation of our intercept and telephone survey, and highlight which of the two might be preferable in light of aspects pertaining to data accuracy, reliability, completeness and so forth.

\subsection{Users' socio-demographic characteristics and travel habits comparison}

As far as gender is concerned no big differences emerge from the two methodologies, with female customers representing the overwhelming majority of the sample surveyed (Table 1). A larger heterogeneity in the results is evident when analyzing the occupation of the interviewees, with a prevalence of students in the on-board survey and retired in the telephone survey. Rather different is also the weight of the category housewives, whilst the steadiest group is represented by the workers, equalling around one-third of the sample for both methodologies.

Telephone interviews appear to be more biased, as the timetable of the survey execution is likely to have excluded the vast majority of students not at home, whilst increasing disproportionately the weight of housewives and retired among the population surveyed. The local transit agency's established schedules in 
Table 1: $\quad$ Gender and age of the sample of users surveyed.

\begin{tabular}{llll}
\hline Variable & Level & Intercept (\%) & Telephone (\%) \\
\hline \multirow{3}{*}{ Gender } & Male & 36.7 & 32.6 \\
& Female & 63.3 & 67.4 \\
& Workers & 29 & 26.6 \\
Occupation & Students & 52.6 & 7.8 \\
& Unemployed & 7.1 & 2.3 \\
& Housewives & 5.6 & 22.9 \\
& Retired & 5.7 & 40.4 \\
\hline
\end{tabular}

telephone surveys and the need to concentrate the calls during specific hours, so to cause little to none disturbance at meal times, are certainly responsible for the overrepresentation of some categories to the detriment of others.

Substantial differences are visible when analyzing average transit behaviours among the samples investigated. Table 2 highlights higher frequencies of use for on-board intercepted customers, with over $70 \%$ declaring to make a daily use of the local public transport system and less than the $8 \%$ admitting to use it occasionally (twice a month or less). Data gathered among telephone owners show lower percentages of high frequency users and over one-fifth of occasional riders.

Such heterogeneity can be easily explained by the kind of riders interviewed in the two surveys, with students making a daily use of the public transport system to go to school and return home and retired travelling mainly for personal matters and shopping (both reasons generally not requiring a daily use of the transport system).

Table 2: $\quad$ Travel habits of the sample of users surveyed.

\begin{tabular}{llll}
\hline Variable & Level & Intercept (\%) & Telephone (\%) \\
\hline & 5/6 times per week & 70.7 & 29 \\
& 3/4 times per week & 13.3 & 13.2 \\
Frequency & $1 / 2$ times per week & 8.7 & 36.6 \\
of use & $1 / 2$ times per month & 1.6 & 16.6 \\
& Occasionally & 5.5 & 4.6 \\
& First timer & 0.2 & - \\
& School & 44.3 & 16.6 \\
Reason for & Work & 22 & 17.2 \\
using bus & Personal matters & 19.5 & 38.4 \\
transport & Home & 7 & 0.3 \\
& Leisure, sport & 4.2 & 8.8 \\
& Shopping & 3 & 18.7 \\
\hline
\end{tabular}




\subsubsection{Non users' socio-demographic characteristics and travel habits}

A key advantage of the telephone survey administration has been represented by the possibility to investigate non users' characteristics and their reasons for avoiding the urban bus transport option. The summary results show an overwhelmingly majority of female among non users, $68.9 \%$ against the $31.1 \%$ of their male counterparts (see Table 3). Significantly, around two-thirds of the population surveyed affirmed to have been reliant on public transport in the past, though having abandoned it more than 10 years before, mainly because of the possess of a car and, with minor relevance, average trips characteristics or availability of someone else's car each time required.

Table 3: $\quad$ Main features of the sample of non users surveyed.

\begin{tabular}{lll}
\hline Variable & Level & Values (\%) \\
\hline \multirow{2}{*}{ Gender } & Male & 68.9 \\
& Female & 31.1 \\
Past use of bus & Yes & 65.7 \\
transport & No & 34.3 \\
Years of non usage & More than 10 & 72.9 \\
& Less than 10 & 27.1 \\
& Car ownership & 60.2 \\
Main reason for not & Short trips & 20.2 \\
using bus transport & Availability of a car & 16.5 \\
& Other reasons & 3.1 \\
\hline
\end{tabular}

\subsection{Customer satisfaction comparison}

Despite having resulted in significant socio-demographic and travel habits differences, the two survey methodologies yield similar results when it comes to determining the level of customer satisfaction and the gaps width between what is expected and what is perceived in terms of service quality. As is visible in Table 4, on-board security is the most important and satisfactory attribute in both cases, whilst the time spent on the bus and the on-board comfort hold a minor relevance for both types of customers contacted. The least satisfactory attributes are represented by the buses cleanness for on-board customers and the buses frequency for telephone customers, even if the differences are far from being relevant.

Similar considerations can be advanced when assessing the gaps width, with values rather similar for the two data gathering methodologies. The only significant differences are related to the attribute information, quite distant from the expectations of the on-board surveyed population and much more in line with the expectations of the telephone surveyed sample.

To sum up, we conclude that the type of survey conducted does not seem to influence users' needs and perceptions, suggesting rather homogeneous judgements regardless of socio-demographic and travel habits differences. 
Table 4: $\quad$ SERVQUAL analysis.

\begin{tabular}{llll|lll}
\hline \multirow{2}{*}{ Attributes } & \multicolumn{3}{c}{ Intercept survey } & \multicolumn{3}{c}{ Telephone survey } \\
& P & A & GAP & P & A & GAP \\
\hline Frequency & 6,97 & 9,35 & $-2,39$ & 7,03 & 9,43 & $-2,40$ \\
Travel time & 7,62 & 8,66 & $-1,05$ & 7,38 & 8,56 & $-1,18$ \\
Punctuality & 7,13 & 9,52 & $-2,39$ & 7,16 & 9,52 & $-2,36$ \\
Cleanness & 6,86 & 9,37 & $-2,51$ & 7,05 & 9,33 & $-2,29$ \\
Comfort & 7,00 & 8,64 & $-1,64$ & 7,46 & 8,92 & $-1,45$ \\
Courtesy & 7,31 & 9,20 & $-1,89$ & 7,57 & 9,22 & $-1,65$ \\
Security & 7,71 & 9,74 & $-2,03$ & 7,63 & 9,64 & $-2,01$ \\
Information & 7,00 & 9,12 & $-2,12$ & 7,45 & 8,85 & $-1,40$ \\
Tickets & 7,45 & 8,99 & $-1,54$ & 7,45 & 9,11 & $-1,66$ \\
\hline
\end{tabular}

\section{Data quality}

The final part of the paper will deal with some issues emerged when surveying the two samples, in an attempt to shed some lights on the most appropriate approach in terms of data quality and accuracy. Both non measurement and measurement errors will be reported, with other influencing factors such as survey checking and burden on respondents also considered. Finally, some considerations will address costs and productivity related issues.

\subsection{Non measurement error}

When analyzing non measurement errors we mainly focused on issues related to sampling bias and non response rates, which, in both cases, strongly suggested the superiority of intercept over telephone surveys. Telephone interviews suffered from a strong bias in the sample surveyed, mainly resulting from the interviews schedule, with an overrepresentation of housewives and retired and a correspondent underrepresentation of the category students (a core component of the local public transport users). A further biasing factor was given by the possibility to sample only users listed in the telephone directories, with less than $60 \%$ of land line owners among the residents in the region of study. Intercept surveys overcome such risks guaranteeing an equal probability for the on-board passengers to be surveyed whilst experiencing the service.

Response rates were much lower for telephone than for intercept surveys. It took an average of almost 4 random phone calls before establishing a contact and just 1 in 3 contacts was represented by a public transport user willing to complete the survey, with a completion rate of $36.3 \%$. On the contrary, the completion rate for the intercept survey reached a significant $86.7 \%$. As stated by Bowling [1], the lowest the response rate the higher the likelihood for the respondent to differ from the non respondent, the higher the study bias, the lower the reliability and generalizability of the survey results. 


\subsection{Measurement error}

The analysis of measurement errors, related to the bias in the answers provided during the questionnaires administration, does not offer a clear-cut picture over which of the two methodologies provides better results. Social desirability bias, the respondents' tendency to modify their answers so to put themselves in a better light vis-à-vis the interviewer, and acquiescence bias, the respondents' tendency to give too positive answers, were somehow registered for both methodologies without a significant difference between the two. Both biases were more evident among eldest respondents, whilst the youngest did seem to be less influenced by the interaction with the interviewer.

\subsection{Survey checking}

Checking the questionnaire administration process has proved to be a strongpoint for the telephone survey, whilst the same accuracy hasn't been possible for the on-board investigation. With reference to the telephone survey, the fact that the interviews were conducted in one single location (the local transit agency headquarters) has enabled a thorough control and the immediate implementation of corrective actions every time problems surfaced. Furthermore, the use of a Computer-Assisted Telephone Interviewing system (CATI) has allowed quicker and more accurate data entry operations. With reference to the intercept survey, random controls on the on-board interviews have been executed by external supervisors. In addition the on-board teams have been organised mixing experienced interviewers with new ones, so to allow the former to supervise the latter and intervene to solve possible problems. This expedient has somehow increased the degree of checking performed during the process, despite not guaranteeing the thoroughness of the telephone methodology. Furthermore, the use of a paper-and-pencil interviewing system (PAPI) in place of a computerassisted personal interviewing system (CAPI) has meant that the data had to be processed twice, making more difficult to control possible errors linked to the double entry system put in place. However, this represents a temporary problem, easily solvable in the near future, when well-trained surveyors will directly elicit information by means of electronic personal devices.

\subsection{Burden on respondents}

The cognitive burden on the respondents has proved to be significantly higher during the telephone survey, with people sometimes providing incoherent answers, showing problems in the correct understanding of some questions, asking to repeat or rephrase them, giving votes without (apparently) much thinking. The lack of a visual contact made also impossible for the interviewer to understand possible difficulties expressed through nonverbal communication means. Such problems have been mostly found among the eldest, which made up a larger share of the sample contacted in the telephone survey. On the other hand, the physical interaction between interviewers and respondents has greatly 
reduced the cognitive burden required to the latter during the intercept survey, with positive effects on the quality and reliability of the data gathered.

\subsection{Monetary and productivity issues in data collection}

Despite not being strictly related to quality matters, it is important to give some insights into cost savings and productivity issues encountered during our investigation, as differences have emerged with much of the existing literature. As previously stated, the superiority of telephone vis-à-vis intercept surveys when it comes to costs and productivity has been frequently pointed out, with Bradburn and Sudman [2] estimating the cost of intercept surveys to be up to twice as high as the cost of telephone surveys and Bonnel and Le Nir [5] explaining these higher expenditures with lower levels of productivity, a mix of low numbers of interviews completed and higher transport and time costs associated to the frequent transfers required for the interviewers to reach the respondents' home. Rather surprisingly, our study produced the opposite outcome, with an overall average number of interviews completed per hour of 2.8 and 1.6 for the on-board and the telephone methodology respectively. Increased levels of productivity for the on-board survey can be primarily explained because of the limited number of transfers among bus, with teams of interviewers working full shifts continuously on a same route. In addition, the personal interaction between them and the respondents, not possible in the other methodology, made more difficult for these latter to refuse to take part in the survey, with rates of completion significantly higher. Such higher productivity levels translated also in lower costs per single interview completed, with each single on-board interview costing around $59 \%$ of a telephone one under the assumptions of equal hourly wages for the two types of interviewers and substantial irrelevance of other costs (as it was in our case).

\section{Conclusions}

The study compared two different survey methodologies with the aim to choose the one more likely to provide transit agencies with reliable socio-demographic, travel habits and customer satisfaction data. As shown in Table 5, both intercept and telephone surveys are characterized by strengths and weaknesses. However, in our case, a lesson that can be drawn is that intercept surveys are to be preferred mainly because of their good levels of sampling coverage and high response rates, both elements pivotal in avoiding biased results of little, if none, usefulness. On the contrary, sampling bias and low response rates severely hamper the validity of telephone surveys, despite the advantages related to real time survey checking operations. In addition, the steady decline registered in land lines ownership in Italy, down to 67.1\% from 84.7\% between 2001 and 2011 (Murgia and Nunnari [26]), is likely to persist, with negative consequences on the already low response rates. This trend will certainly increase sampling bias problems in telephone surveys, thus further lowering data quality and reliability. 
Another consideration that can be advanced is that telephone surveys shouldn't yet be discarded entirely, as they help reaching non users and collect relevant information on the reasons behind their choice to rely on other modes of transport, besides their travel patterns. Therefore, the two methodologies might be used in conjunction to gather relevant data on users and non users and provide public transport operators with relevant information, exploitable to enhance customers retention and non-customers acquisition.

Table 5: $\quad$ Comparison of intercept and telephone surveys.

\begin{tabular}{|c|c|c|}
\hline & Main strengths & Main weaknesses \\
\hline 冚 & $\begin{array}{c}\text { Good sampling coverage at route level } \\
\text { Good socio-demographic sampling coverage } \\
\text { High data reliability and accuracy } \\
\text { Ease of clarifying question } \\
\text { High response rate } \\
\text { Low cognitive burden } \\
\text { Lower costs }\end{array}$ & $\begin{array}{l}\text { Less accurate data loading process } \\
\text { Social desirability/acquiescence bias } \\
\text { Low survey checking }\end{array}$ \\
\hline$\frac{\mathscr{0}}{\frac{\partial}{0}}$ & $\begin{array}{l}\text { More accurate data loading process } \\
\text { High survey checking } \\
\text { Good geographic sampling coverage }\end{array}$ & $\begin{array}{c}\text { Poor socio-demographic sampling coverage } \\
\text { Low response rate } \\
\text { Lower data reliability and accuracy } \\
\text { Social desirability/acquiescence bias } \\
\text { High cognitive burden } \\
\text { Lower study legitimacy } \\
\text { Higher costs }\end{array}$ \\
\hline
\end{tabular}

However, given the mentioned decrease in land lines use and the concurrent ageing of their holders, future studies might address the feasibility of alternative survey modes, mainly mobile phones or web based, to elicit information on those who decide to rely on private transport for their transfers. Indeed, the recent upward trend recorded in Italy in the use of mobile phones and the internet let us believe that, despite present statistics still showing the opposite, the two tools will soon be characterized by higher penetration and usage rates than land lines.

Finally, the existing literature on surveys in public transport has often pointed out how different methods might be skewed towards some sub samples of the overall population surveyed, with the eldest more likely to be overrepresented in telephone surveys, the youngest in internet surveys, the more educated in self completion mail surveys and so on. Hence, a possible further line of research might address the issues of adopting mixed data collection techniques as a tool to maximize sample representativeness, response rates and reduce possible biases associated to the adoption of a single methodology. The positive effects on data quality, reliability and results generalizability will certainly boost the applicability and usefulness of surveys in public transport. 


\section{References}

[1] Bowling, A., Mode of questionnaire administration can have serious effects on data quality. Journal of Public Health, 27 (3), pp. 281-291, 2005.

[2] Bradburn, N. M. \& Sudman, S., (eds). Polls and Surveys: Understanding what They Tell Us, Jossey-Bass: San Francisco, 1988.

[3] Shih, T. H. \& Fan, X., Comparing response rates from Web and mail surveys: A meta-analysis. Field Methods, 20 (3), pp.249-271, 2008.

[4] Lozar Manfreda, K., Bosnjak, M., Berzelak, J., Haas, I. \& Vehovar, V., Web surveys versus other surveys modes: A meta-analysis comparing response rates. International Journal of Market research, 50 (1), pp. 79104, 2008.

[5] Bonnel, P. \& Le Nir, M., The quality of survey data: Telephone versus face-to-face interviews. Transportation, 25 (2), pp. 147-167, 1998.

[6] Sturges, J. E. \& Hanrahan, K. J., Comparing telephone and face-to-face qualitative interviewing: A research note. Qualitative Research, 4 (1), pp. 107-118, 2004.

[7] de Leeuw, E. D. \& Hox, J. J., Internet surveys as part of a mixed-mode design (Chapter 3). Social and behavioural research and the internet: Advances in applied methods and research strategies, ed. Das, J. W, M., Ester, P. \& Kaczmirek, L., Taylor and Francis: New York, pp. 45-76, 2010.

[8] Endemann, P., Review of travel survey usefulness. Paper presented at $12^{\text {th }}$ World Conference on Transport Research (WCTR), Lisbon, 2010.

[9] Stopher, P. R., The travel survey toolkit: Where to from here? Paper presented for the $8^{\text {th }}$ International conference on travel survey methods, Annecy, 2008.

[10] Inbakaran, C. \& Kroen, A., Travel surveys - Review of international survey methods. Paper presented at the Australasian Transport Research Forum, Adelaide, 2011.

[11] Sharp, J. \& Murakami, E., Travel surveys: Methodological and technologyrelated considerations. Journal of Transportation and Statistics, 8 (3), pp. 97-113, 2005.

[12] Bonnel, P., Postal, telephone and face-to-face surveys: how comparable are they? (Chapter 12). Transport Survey Quality and Innovation, ed. Stopher, P.R. \& Jones, P.M., Pergamon: Oxford, pp. 215-237, 2003.

[13] Moritz, G. \& Brög, W., Redesign of the Dutch travel survey: Response improvement. Paper presented at Transportation Research Board (TRB) on personal travel: The long and short of it, Washington D.C., 1999.

[14] Morris, J., \& Adler, T., Mixed mode surveys. (Chapter 13). Transport Survey Quality and Innovation, ed. Stopher, P. R. \& Jones, P. M., Pergamon: Oxford, pp. 239-252, 2003.

[15] Bayard, C. \& Bonnel, P., Combining web and face-to-face in travel surveys: Comparability challenges? Transportation, 39 (6), pp. 1147-1171, 2012. 
[16] Eboli, L. \& Mazzulla, G., A methodology for evaluating transit service quality based on subjective and objective measures from the passenger's point of view. Transport Policy, 18 (1), pp. 172-181, 2011.

[17] Lai, T. W. \& Chen, C. F., Behavioral intentions of public transit passengers - The roles of service quality, perceived value, satisfaction and involvement. Transport policy, 18 (2), pp. 318-325, 2011.

[18] Thompson, K. \& Schofield, P., An investigation of the relationship between public transport performance and destination satisfaction. Journal of Transport Geography, 15 (2), pp. 136-144, 2007.

[19] Fellesson, M. \& Friman, M., Perceived satisfaction with public transport service in nine European cities, The Journal of Transportation Research Forum, 47 (3), pp. 93-103, 2008.

[20] Friman, M., Edvardsson, B. \& Gärling, T., Frequency of negative critical incidents and satisfaction with public transport services. Journal of Retailing and Consumer Services, 8 (2), pp. 95-104, 2001.

[21] Too, L. \& Earl, G., Public transport service quality and sustainable development: A community stakeholder perspective, Sustainable Development, 18 (1), pp. 51-61, 2010.

[22] Cantwell, M., Caufield, B. \& O’Mahony, M., Examining the factors that impact public transport commuting satisfaction, Journal of Public Transportation, 12 (2), pp. 1-21, 2009.

[23] Tyrinopoulos, Y. \& Aifadopoulou, G., A complete methodology for the quality control of passenger services in the public transport business. European Transport, 38, pp. 1-16, 2008.

[24] Beirão, G. \& Sarsfield Cabral, J. A., Understanding attitudes towards public transport and private car: A qualitative study. Transport Policy, 14 (6), pp. 478-489, 2007.

[25] Barabino, B., Deiana, E. \& Tilocca, P., Measuring service quality in urban bus transport: A modified SERVQUAL approach, International Journal of Quality and Service Sciences, 4 (3), pp. 238-252, 2012.

[26] Murgia, M. \& Nunnari, A., Improving the quality of data collection: Minimum requirements for generalised software independent from the mode, Paper presented at the Conference of European Statisticians Seminar on New Frontiers for Statistical Data Collection, Geneva, 2012. 